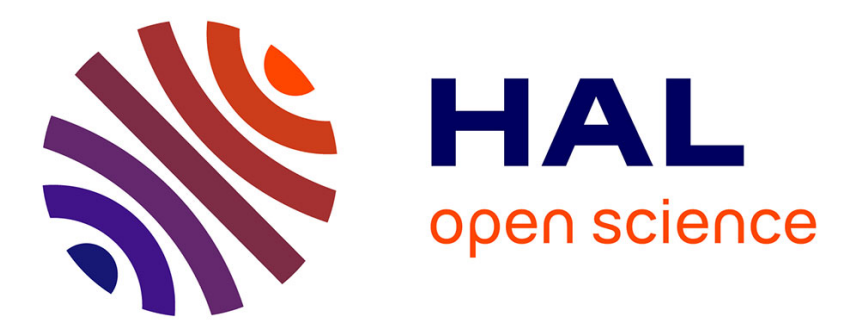

\title{
Un jeune Bacchus, ornement de trépied, provenant de Gaule, à la Walters Art Gallery de Baltimore Dorothy Kent Hill
}

\section{To cite this version:}

Dorothy Kent Hill. Un jeune Bacchus, ornement de trépied, provenant de Gaule, à la Walters Art Gallery de Baltimore. Gallia - Fouilles et monuments archéologiques en France métropolitaine, 1952, 10, pp.31-42. hal-01936189

\section{HAL Id: hal-01936189 \\ https://hal.science/hal-01936189}

Submitted on 6 Feb 2020

HAL is a multi-disciplinary open access archive for the deposit and dissemination of scientific research documents, whether they are published or not. The documents may come from teaching and research institutions in France or abroad, or from public or private research centers.
L'archive ouverte pluridisciplinaire HAL, est destinée au dépôt et à la diffusion de documents scientifiques de niveau recherche, publiés ou non, émanant des établissements d'enseignement et de recherche français ou étrangers, des laboratoires publics ou privés.

\section{(ㅇ)(1) $\$$}

Distributed under a Creative Commons Attribution - NonCommercial - NoDerivatives| 4.0 


\title{
UN JEUNE BACCHUS, ORNEMENT DE TRÉPIED, PROVENANT DE GAULE, A LA WALTERS ART GALLERY DE BALTIMORE
}

\author{
par Miss Dorothy K. HiLL
}

Les objets antiques une fois aperçus et puis perdus peuvent réapparaître après des générations, sans qu'on les reconnaisse tout d'abord. C'est le cas du Bacchus romain ici reproduit (fig. 1), qui appartenait à la collection du regretté Henry Walters, de Baltimore. Je me propose de démontrer que cette statuette est celle-là même qu'ont déjà fait connaître certaines publications : trouvée dans le sol de la Gaule, elle mérite d'être ici publiée à nouveau.

Elle a été décrite par Coutil qui l'a dessinée en 1894 et publiée en 1895 (fig. 2) ${ }^{1}$ : "La statuette représentait un Bacchus... Ce Bacchus est nu, orné de la nébride, qui lui couvre la poitrine et les épaules. Cette nébride est remarquable en ce qu'elle est incrustée de filets d'argent, chose assez rare... L'ouverture carrée pratiquée dans le socle et la soudure placée entre les deux épaules, tendent à prouver que cette statuette faisait partie d'un autel ou d'une décoration quelconque. Il semble... que la statue n'ait été faite que pour être vue de face ". L'auteur omet sur l'illustration, sans donner de raison, la nébris qu'il a décrite si soigneusement : de là vient la confusion, bien superflue, qui s'est produite à propos de cette statuette. De plus, il ne dit pas comment il a pu fournir une reproduction même rudimentaire d'un objet depuis longtemps inaccessible.

Coutil se réfère à une communication de L. Passy à la Société des Antiquaires de France, ainsi rédigée dans le Bulletin ${ }^{2}$ : "Dans le courant de l'année dernière, un cultivateur du Thuit (commune de l'arrondissement des Andelys, Eure)... découvrit deux squelettes. La tête du plus petit reposait sur le bras du plus grand; auprès de la tête il trouva la statuette que j'ai l'honneur de

(1) L. Coutil, Département de l'Eure. Archéologie gauloise, gallo-romaine, franque et carolingienne (1895) I. Arrondissement des Andelys, pp. 41 sq., pl. face à p. 68, signée « Coutil 1894 》.

(2) Bulletin de la Société Impériale des Antiquaires de France, 1862, séance du 22 janvier, pp. 33-35. 
présenter à la Société; plus bas, vers les pieds, le petit vase, deux médailles et un anneau en argent. La statuette est l'objet capital de la découverte; elle représente un Bacchus; les cheveux abondants, partagés sur le front et relevés

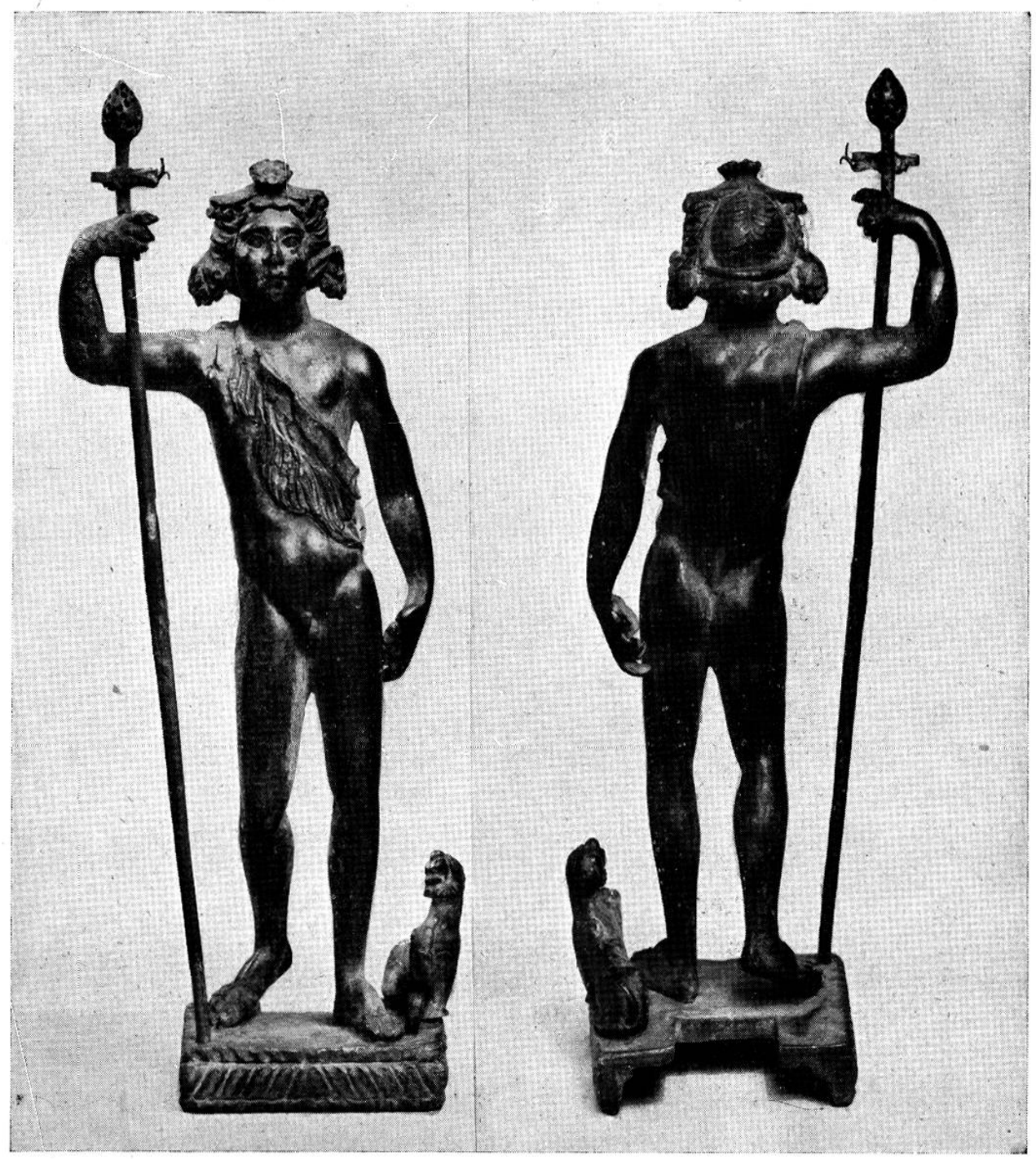

Fig. 1. - Bacchus de la Walters Art Gallery, Ballimore, U.S. $\Lambda$. (haut.: $0^{\mathrm{m}} 163$ ).

sur les tempes, sont rassemblés derrière la tête comme ceux d'Apollon. La coiffure se compose d'une fleur placée sur le front en forme de diadème et de grappes de raisin qui tombent des deux côtés de la figure. Bacchus est nu; il est orné de la nébride qui, cette fois, n'est pas une peau de panthère, mais 
une peau de chevreuil. Cette nébride a ceci de remarquable, qu'elle est plaquée d'argent, et l'on sait par d'autres exemples que les statuettes religieuses ornées de placages d'argent étaient l'objet d'une vénération particulière. Bacchus a les pieds nus; la main droite est levée, elle tenait évidemment un thyr'se qui devait entrer dans un trou pratiqué dans le socle... L'ouverture carrée pratiquée dans le socle et la soudure placée entre les deux épaules tendent à prouver que cette statue faisait partie d'un autel ou d'une décoration quelconque... On peut noter que l'anneau en argent a été jadis octogone... Les deux médailles sont d'Antonin et de Posthume. »

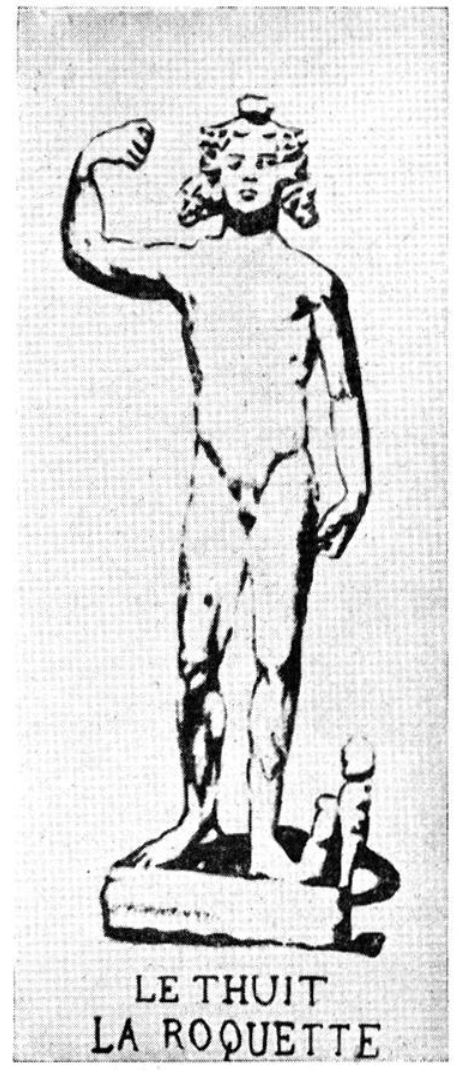

FiG. 2. - Dessin de Coutil (1894).

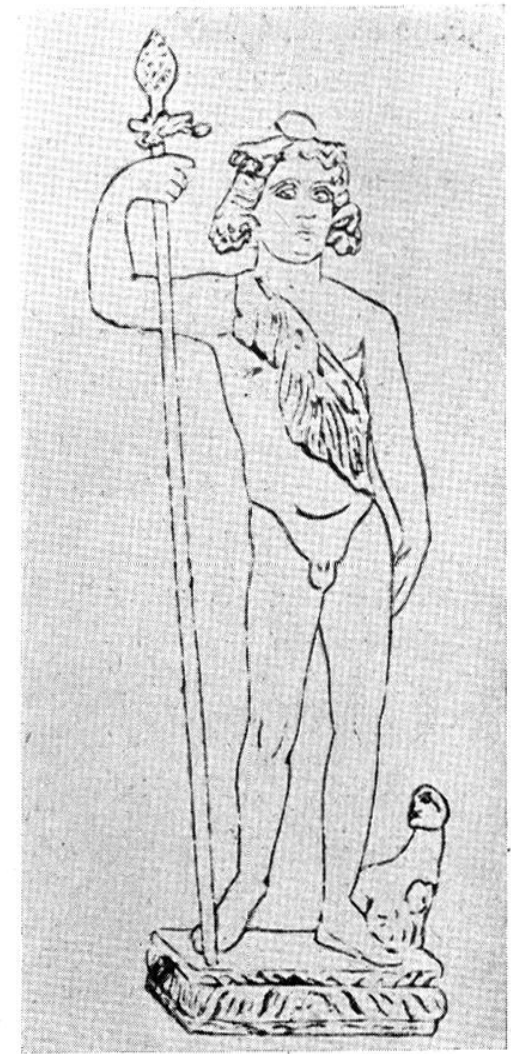

FIG. 3. - Dessin publié par S. Reinach (1904) d'après la photographie du catalogue Forman.

Dans la discussion qui suivit, M. Chabouillet suggéra qu'étant donnée la fleur qui orne le diadème, il s'agissait du génie de Bacchus plutôt que du dieu lui-même, et M. Bordier identifia avec le symbole celtique S l'objet placé sous la patte de la panthère, citant un objet analogue sous le sabot d'un cheval en bronze. Dans le Supplément à son répertoire qu'il publia en 1921, 
Coutil donna quelques précisions supplémentaires sur l'histoire de la statuette, qui figura à " l'Exposition archéologique d'Evreux en 1864, sous le n 1614 ; elle appartenait alors à M. Théophile Montier, du Thuit, qui l'avait trouvée en 1861; elle fut achetée par Charaet, marchand d'antiquités du Pecq, près Saint-Germain-en-Laye "): aucune allusion à sa dernière destination non plus qu'aux conditions d'exécution du dessin, qui avait été entre temps reproduit par S. Reinach ${ }^{3}$.

D'autre part, l'histoire du bronze ici publié est très facile à retracer: il a encore son étiquette imprimée, où on lit " National Exhibition of Works of Art. Leeds 1864. W. W. Forman, Proprietor ". Ce collectionneur anglais l'avait acquis avant 1868, et le garda jusqu'à la vente en 1899 de sa collection, dont le catalogue reproduit une bonne photographie, identique à celle de notre figure 1 (à gauche) ${ }^{4}$. M. Walters l'acquit vraisemblablement à ce moment, ou peu après, et l'inclut dans la collection qu'il laissa à la ville de Baltimore quand il mourut en 1931. La photographie du catalogue de la vente Forman fut à son tour reproduite par Reinach sous forme de dessin (fig. 3$)^{5}$.

Il résulte de tout cela que la statuette Forman et celle trouvée au Thuit ne font qu'une : le fait a échappé à Coutil et à Reinach, et à moi-même quand je rédigeai le catalogue des bronzes de la Walters Art Gallery ${ }^{6}$. Qu'il suffise de souligner: que la nébris est décrite par Passy et par Coutil, bien qu'elle manque sur la planche publiée par ce dernier; que le rectangle découpé sur l'arrière de la base et les marques d'une attache au milieu du dos se voient sur le bronze exactement comme on les décrit; que les détails, le style et les dégradations (par exemple l'index de la main droite) sont identiques sur l'objet comme dans la description; et que le trou mentionné pour la base du thyrse existe encore au coin avant droit du socle.

Le pedigree est donc reconstitué comme suit: trouvé entre le Thuit et La Roquette en 1861; discuté à la Société des Antiquaires en 1862; exposé à Evreux en 1864; acquis successivement par Montier, Charaet, Forman et exposé à Leeds en 1868; dessiné par Coutil sans la nébris en 1894 et publié par lui en 1895 (fig. 2); reproduit par Reinach en 1897; publié et vendu à la vente Forman en 1899; acquis par Henry Walters; reproduit par Reinach d'après le catalogue Forman en 1904 (fig. 3); republié dans le Supplément de Coutil, sans illustration additionnelle, en 1921.

(3) S. ReINACH, Répertoire de la statuaire grecque et romaine, II, 121, 9 (1897; 2e éd., 1908).

(4) The Forman Collection, London, Sotheby, IVilkinson and Hodge, 19 juin 1899, p. 17, $\mathrm{n}^{\circ}$ 106, pl. VII. L'auteur de ce catalogue de vente vit la ressemblance avec Reinach, II, 121, 9.

(5) O. l., III, 31, 5 (1904, 2e éd. 1920).

(6) Dorothy Knnt HuL, Catalogue of Classical Bronze Sculpture in the Walters Art Gallery, Baltimore, 1949, p. 24, n 42, pl. 14. 


\section{Description.}

La statuette mesure $0^{\mathrm{m}}, 163$ de hauteur, non compris le thyrse, y compris le socle.

Le bronze est franchement jaune. La nébris est une fine plaque d'argent isolée: pour la maintenir en place, le bord supérieur de la nébris de bronze est recourbć et martelé tout le long de l'épaule droite jusqu'à la hanche gauche. Les yeux sont d'argent, les pupilles creuses étaient remplies de menues petites parcelles blanchâtres dont une subsiste à l'œil gauche. Le bronze a été débarrassé de la terre qui l'a recouvert, sauf aux endroits les moins accessibles: dans l'entre-jambes, aux aisselles et sous la masse des cheveux. La surface dans son entier est polie et recouverte d'une belle patine verte présentant peu de lacunes: une entaille profonde au menton, des stries horizontales au côté droit (notamment sur l'abdomen et, très profonde, à la cuisse), une profonde coupure à travers les doigts du pied droit. La plaie du menton peut être moderne, mais non les autres, car la patine, bien qu'assez amincie par endroits pour laisser transparaitre le jaune brillant du bronze, y existe dans toutes les coupures, parfois même très épaisse. Il va de soi que ces dégradations n'ont pu être effectuées pendant que le côte droit était protégé par le thyrse. Elles résultent manifestement de coups répétés donnés'à partir du haut et de la droite.

Autant qu'on sache, le thyrse, qui n'était pas en place quand la statuette a été montrée et discutée en 1862, a été ajouté entre cette année et 1868 . Toutefois, il n'est pas entièrement moderne, comme je l'ai dit dans mon catalogue: la partie au-dessus de la main est d'un métal récent, recouverte d'une fausse patine, et fixée à la main avec du plâtre; mais la hampe est d'un métal ancien, avec une bonne patine, et introduite à travers la main jusque dans la base. Bien que pour des raisons purement techniques, je sois tentée de nier que le thyrse appartienne originellement à la statuette, je dois reconnaître qu'il est difficile de suggérer l'insertion d'un autre attribut dans la main droite levée, légèrement fermée, le pouce touchant l'extrémité du $3^{\mathrm{e}}$ et du $4^{\mathrm{e}}$ doigt, l'index ètendu et toute la main tournée le dessus vers l'avant. Le plâtre qui la remplit rend des plus difficiles la détermination de l'objet tenu: une grappe de raisins, un rhyton ou un autre vase ne sont pas impossibles.

La main gauche est intacte, le poignet plié vers l'extérieur et vers l'avant. Elle est fermée, le pouce touchant le bout du $3^{3}$ doigt, l'index étendu. Un trou circulaire et calibré remplace l'orifice naturellement irrégulier séparant le pouce et les autres doigts. Le $3^{\text {e }}$ et le $4^{\text {e }}$ doigts sont légèrement creusés sur le dessus pour faire place au bord de l'attribut, qui devait être fait d'une pièce 
séparée, probablement un vase à anses rubanées, canthare ou œnochoé, tenu négligemment de telle sorte que le contenu s'en échappât vers l'avant ?. Une grappe, bien que converiant à la tête levée de la panthère, est invraisemblable à cause de l'attitude de la main presque fermée.

Une courte baguette ronde sous l'arrière-train de la panthère, fondue avec elle, passe à travers un trou de la base et est fixée au marteau sur sa face inférieure comme un rivet. L'extrémité aplatie étant bien patinée, l'attache paraît antique. La base, si épaisse que l'intérieur en est à peine vide, a été fondue en même temps que la statuette, comme le montrent le dessous du pied droit et les bords du pied gauche. L'évidement carré à l'arrière de la base est patiné et ne peut être moderne. Plus frappant encore est le fait que le dessus moutonné, en arrière de la statuette, est aussi recouvert d'un vert lisse.

\section{Les supports de pieds de table.}

L'évidement de la base et la marque d'attache qui est directement audessus dans le dos de la statuette font directement supposer que l'objet était la partie inférieure du pied mince d'une table ou d'un trépied. On peut \&omparer le très jeune "Génie bacchique " du Musée de Saint-Germain: cette petite divinité est debout sur une base peu élevée, et entourée d'une guirlande de lierre (il en existe une réplique très semblable dans le commerce) ${ }^{8}$. Derrière, touchant son dos, est un montant de bronze carré et creux. Au même Musée se trouve encore la statuette d'un Bacchus un peu plus âgé, tout droit, un montant du même genre attaché à son dos, mais ne descendant pas jusqu'à la base ${ }^{9}$. (Les moulures horizontales sur les bases de ces deux objets, avec les hachures verticales et obliques maladroitement faites, sont comparables aux moulures de ia base de notre statuette). Il y a d'autres exemples de statuettes en bronze jouant le rôle de pieds de table amovibles: un Hercule trouvé en Allemagne, un enfant tenant un oiseau à la Bibliothèque Nationale, et une Tyché d'Antioche dans une collection espagnole ${ }^{10}$. Vraisemblablement

(7) Entre autres exemples : Espérandieu, Recueil gén., X, p. 103, en bas; A. Garcia y BelLino, Esculturas Romanas de España y Portugal, 1949, pp. 96 sq., $\mathrm{n}^{\circ} 82$, pl. 71, ou dans Ars IIispaniae, II, p. 93, fig. 67; Gazette des Beaux-4rts, sér. III. 38, 1896, I, p. 260 ou Mon. Ant., VIII, 1898, p. 287, fig. 22; HiLl, o. l., p. 23, $\mathrm{n}^{\circ} 41$, pl. 14; PERDinzET, Bronzes grecs d'Egypte de la Collection Fouquet, 1911, pl. VI.

(8) S. ReInach, Antiquités nationales. Description raisonnée du Musée de Saint-Germain-enLaye. Bronzes figurés de la Gaule romaine, pp. 245 sq., $\mathrm{n}^{n}$ 237, et Rep. Slat., II, 126, I; cf. ibid., IV, $70,3$.

(9) Reinach, Bronzes figurés, p. 87, n 79 . Autrefois dans la Collection Hoffman, Catalogue de vente, 1888, p. $140, n^{\circ} 511$ (non reproduit; «décor de meuble, trouvé en Gaule»).

(10) Espérandieu, Recueil général... de la Germanie rornaine, pp. 338 sq., no 528 ; Archivo Esp. de Arch., 21, 1948, pp. 64 sq., fig. 12, and A. GARCia J Bellido, Esculturas Romanas, 1949, 
les parties supérieures des montants étaient en une autre matière, bois ou fer, mais la construction demande seulement qu'elles soient amovibles. Notre Bacchus diffère de certains d'entre eux seulement par l'exécution complète du dos, et presque aucunement des autres. Quant aux coupures subies par son côté droit, elles peuvent, précisément, être dûes à l'usage fait du meuble qu'il décorait.

L'emploi de ces figures de support n'est pas limité aux meubles métalliques. Une ample série de pieds de table en marbre, tous ornés des mêmes sujets, est connue depuis les temps hellénistiques jusqu'aux premiers temps chrétiens ${ }^{11}$. Bien que la plupart, sinon tous, soient des pieds de milieu, uniques, et bien que les figures sculptées soient grandes par rapport aux autres parties de la table, la ressemblance avec les supports de bronze est frappante ${ }^{12}$.

Le nombre des bronzes qui, comme notre Bacchus, servaient de pieds de tables et de trépieds est trop faible pour que l'on puisse dater ou reconstituer complètement cette série de meubles, mais ils rappellent un groupe étendu de tables pliantes ou de trépieds que l'on connaît beaucoup mieux ${ }^{13}$. Parmi les plus représentatifs de ce groupe, on doit citer toult d'abord un fragment de pied de table trouvé à Tvarditza (Bulgarie) qui a pour base un satyre à genoux sur une plinthe carrée, le pied lui-même étant inséré dans une attache qui sort de son dos ${ }^{14}$. Il y a aussi le trépied d'Industria (Italie), avec la partie supérieure d'un Silène émergeant de feuilles au-dessous du pied d'animal habituel, et le pied du trépied sortant à son tour d'un support attaché à son dos ${ }^{15}$. Il faut citer aussi la table ì quatre pieds de Sackrau (Allemagne), dont les pieds sont des pattes mais comportant en haut de leur face extérieure

pp. 171 sq., $\mathrm{n}^{\circ}$ 187, pl. 141; Babelon et Blanchet, Catalogue des bronzes antiques de la Bibliothèque Nationale, 1895 , pp. 232 sq., n 557.

(11) Lehmann-Hartleben, Bellepheron und der Reiterheilige, Röm. Mitt., XXXVIII-XXXIX, 1923-4, pp. 264-280; v. en particulier son n० 21, Copenhagen, Antike Kunstvaerker I, 1907, pl.

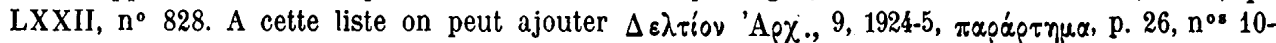
12, p. 23, fig. 14-16; Jahreshefte, XI, 1908, p. 161, fig. 53 et 54; B.C.H., XXXIII, 1909, pp. 262 sq.. n० 9 , fig. 7.

(12) Comparez Richter, Ancient Furniture, fig. 328 avec les fig. 322-326; la fig. 327 a des pieds de bronze aussi grands que les pieds de marbre.

(13) Bibliographie générale : Schwendemann, Jahrb. d. Arch. I, XXXVI, 1921, pp. 107 sq.; WUilleumier, Mél. Ec. Fr. Rom., 45, 1928, pp. 128-149; G. ERdelyi, Archaeologiai Ertesito, 45. 1931, pp. 1 sq.; et ma note de l'A.J.A., 55, 1951, pp. 148 sq., et 345-347, pp. 38 sq.

(14) Arch. Anz., 1915, col. 227-8, fig. 8, 9. Je pense qu'il s'agissait d'une table à quatre pieds, non d'un trépied, parce que le crochet se présente comme sur les pieds postérieurs de la table de Sackrau.

(15) Atti della Societh piemontese di Archeologia e Belle Arti per la Provincia di Torino, III. 1880, p. 101, pl. XVI; P. BArucchI, Memorie della Classe di Scienze morali, R. Accademia delle sicienze di T'orino, XXXIII, 1829, pp. 138-150 et pl. face à la p. 138; Giornale scientifico, letterario e delle arti (Societa di Torino), I. IV, 1789, pp. 133 sq.; ReiNACH, Rép. Stat., II, p. 388, 8; II, p. 705,3 , et II, p. 62,11 . 
un petit Bacchus portant la nébris, la main droite levée à la hauteur de la tête, et balançant une grappe de raisins au bout de son bras droit abaissé ${ }^{16}$.

\section{Le type du Bacchus.}

La ressemblance générale avec ce groupe de trépieds et de tables pliants est importante car, si nous étudions sa pose et son style, nous serons ramenés presque constamment au même groupe d'objets mobiliers.

Auparavant, reportons-nous à l'unique objet qui puisse être mis exactement en parallèle avec notre bronze: c'est une statuette du Musée de Zagreb qui, comme la nôtre, a le corps d'un jeune homme et la tête d'un enfant en bas âge, n'ayant pas plus d'un an. Les pieds et la main droite manquent, mais la pose générale est identique, le genou droit étant légèrement plié et en avant, le bras droit levé, le gauche abaissé avec la main repliée commé pour tenir un objet ${ }^{17}$. La nébris a la même surface ridée et la même bordure festonnée, le visage a le même menton fort et les joues pleines, et la tête offre les mêmes curieuses protubérances: une fleur au sommet, posée sur des feuilles étalées, avec des grappes pendant de chaque côté des oreilles. N'était la distance qui sépare les lieux des deux découvertes, en France et en Yougoslavie, on serait tenté d'attribuer les deux statuettes à un même meuble, et il n'existe pas d'autre objet dont on pourrait en dire autant.

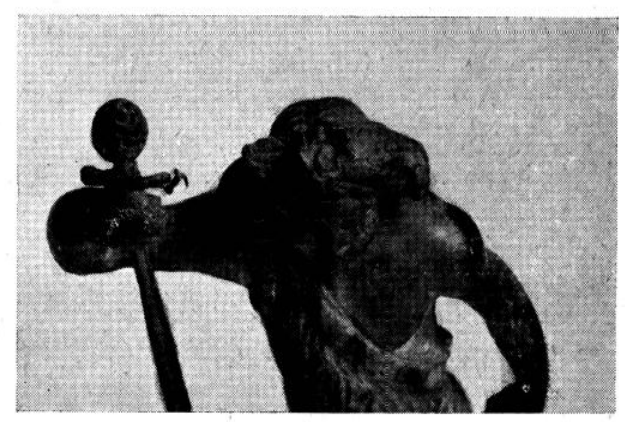

Fig. 4. - Partie supérieure du Bacchus : la coiffure.

(16) Grempler, Der Fund von Sackrau I, 1887, p. 5, pl. III; WILlens, Neue Untersuchungen über die römische Bronzeindustrie von Capua und von Niedergermanien, 1907, pp. 96 sq., fig. 56; ReinaCh, Rép. Stat., III, p. 34, 5; Wuilleumer, Mél. Ec. Fr. Rom., 1928, pp. 143 sq., n 28. Comparez aussi B. de Montfaucon, L'antiquité expliquée et représentée en figures, I, pl. 146, 4 and I, p. 235; Antiquity Explained, I, 1721, II e partie, livre I, p. 148 and pl. face à la p. 147, n० 7; Reinack, Rép. Stat., II, p. 122, 2.

(17) BRusšmid, Hrvatsko arheolosko društvo in Zagreb, Viestnik (Vjesnik) XIII, 1913-14, p. 225: REINACH, Rép. Stat., 5, p. 45, n 4. 
Bien que les statuettes ayant ce visage et ces ornements de coiffure soient si rares, l'élément supérieur habituel des trépiedss et des tables est un buste de même type, que l'on décrit diversement comme un Bacchus ou une Ménade. Tous ces petits personnages ont un visage auquel des joues et un menton anormalement ronds ainsi qu'un aplatissement général confèrent un caractère exagérément enfantin. De grandes grappes sur les oreilles et une fleur posée sur deux feuilles dont les extrémités ourlées se relèvent, donnent à la tête un contour curieux, irrégulier ${ }^{18}$. De tous les bustes de trépieds et de tables, c'est celui de la table de Tvarditza qui ressemble le plus au nôtre. Il a les deux mêmes boucles plates sur le front et, me semble-t-il, le même rendement des grappes par cercles à dépressions centrales. Les raisins, bien qu'ils ne soient jamais des ornements courants de la coiffure, apparaissent sur certaines statues de Bacchus comme d'autres divinités ${ }^{19}$, mais la fleur et les deux feuilles forment une combinaison qui paraît être réservée aux objets précités (fig. 4). Comme ornements comparables, on voit des scarabées ailés, des disques solaires ailés, la couronne impériale laurée à médaillon central et dont les feuilles pointent dans la direction opposée, et même certains arrangements de fleurs et de touffes de cheveux, - mais aucun n'est identique. Tout en étant inca-

(18) Sur un trépied de la Bibliothèque Nationale (inédit; v. plus bas, p. 41); probablement sur celui du Gaire, bien que la fleur seule se voie clairement sur la reproduction (infra, n. 20); sur la table de Sackrau (supra, n. 16) et la lable de Tvarditza (supra, n. 14); probablement le trépied de Bavai, Bull. de l'Institut Arch. Liégeois, XXXII, 1902, pi. B après la p. 342, fig. 6.

De nombreux éléments détachés, avec les crochets indiquant qu'ils appartiennent à de tels Irépieds et tables, sont identiques à notre Bacchus, dont: Babelon et Blanchet, Cat. des bronzes ant. de la Bibl. Nationale, pp. 212 sq., $\mathrm{n}^{\circ \mathrm{s}} 486$ et 487 (cf. aussi 485, grappe mais ni fleur ni feuilles); BIEBER, Die antiken Skulpturen und Bronzen in Cassel, p. 79, n 297, pl. XLVII; SCHUMacher, Beschreibung der Samnlung antiker Bronzen (Grossherzogliche Vereinigte Sammlungen zu Karlsruhe), p. $74 . \mathrm{n}^{\circ} 417$, pl. VII, n 8. V. aussi MonTfaucon, Antiquité Expliquée, vol. II, II partie, livre III, face.å la page 86 (dessin d'un trépied complet). Pour de semblables éléments de couronnement présentant d'autres sujcts, v. Ej)GaR, Greek Bronzes (Catalogue général, Musée lu Caire), pl. VII.

V. aussi une attachc avec la fleur mais une guirlande de feuilles différente, Arch. Zeit., 1883. col. 177 sq.; une avec raisins mais sans fleur ni feuilles, Opus Arch., 5, 1948, p. 36, n० 76, pl. XIX; et le trépied d'Industria (supra, n. 15) qui a la fleur mais ni les grappes ni les feullles, autant que j'en puisse juger.

Pour la fleur portée par d'autres sujets, v. REINACH, Bronzes figuress, pp. 123 sq., 125 (Hercule); Pendnizet, Les terres cuites grecques d'Eyypte de la Collection F'ouquet, pp. 62 sq. nos 160-162, pl. L (Nil).

Pour le véritable enfant Bacchus v. par exemple, Espérandreu, Recueil général, X, p. 242, $n^{\circ} 7646$ et IV, p. 333, n 3371 , ou Mon: Piot, III, 1896, pl. V.

(19) Par exemple, un Bacchus, mais d'un autre type, ReinaCH, Rép. Stat.y V, p. 50, n 7; aussi De RIDDER, Bronzes antiques du Louvre, I, $\mathrm{n}^{\circ} 1061$, pl. 62 (feuille dressée au milieu de la tête); un sarcophage de la Walters Collection, Lehmann-Hartleben et Olsen, Dionysiac Sarcophagi in Baltimore, 1942, fig. 7 et le sarcophage Cassall, ibid.,fig. 40 et Brunn-Bruckmann, 410. Mais v. aussi la grande tète du Louvre, identifiée diversement, mais sûrement pas Bacchus, Gusman, L'art décoratif de Rome, II, pl. 80 . 
pable de l'interpréter, je tiens pourtant que l'emploi limité de cet ornement indique une étroite connection entre notre Bacchus et les trépieds et tables. Dans une étude précédente, j'avais refusć dc datcr deux trépieds de Nubie du $v^{\circ}$ ou $\mathrm{vr}^{\mathrm{e}}$ siècle de notre ère, et des éléments détachés de trépieds à l'époque de Pompéi, dans le premier cas à cause du style, dans le second à cause de la construction du meuble ${ }^{20}$. Le groupe entier des tables et trépieds à pieds droits pliants avec crochets au sommet appartient, selon moi et d'autres auteurs, au milieu de la période impériale. Cette date n'est pas sans conséquence pour notre statuette.

Regardons maintenant la panthère du Bacchus Walters et voyons si elle n'a pas quelque ressemblance avec les têtes de panthère qui surmontent des poignées de plusieurs de ces trépieds, et plus encore avec les avants de panthère qui apparaissent à côté de Bacchus au sommet de chaque pied antérieur de la table de Sackrau. Remarquez les taches qui, sur la panthère complète, sont faites d'un coup d'instrument d'une grandeur disproportionnée. C'est à cause de la ressemblance du style et des taches que je suis certaine que la panthère appartient à notre Bacchus, bien qu'elle ait pu être changée de place de façon à occuper sa position actuelle ${ }^{21}$.

L'étude du corps n'apporte rien à notre sujet. Malgré ses proportions d'ensemble " classiques ", la statuette ne reproduit aucun chef d'œuvre grec reconnaissable: ce n'est qu'un pastiche postérieur aux grandes productions de la Grèce. A la haute époque romaine, il existait déjà pour Bacchus une embarassante diversité de types mélangés: on peut conclure. seulement que notre statuette n'est pas antérieure à l'époque romaine. Une tradition dérivée de Callistrate rapporte que Praxitèle a créé un Bacchus portant la nébris ${ }^{22}$, et l'on a tenté de reconnaître le type praxitélien dans un bronze du Louvre, mais cette théorie n'a pas été acceptée par tous ${ }^{23}$. Même si la pose et la nébris ont quelque chose de vaguement praxitélien, le modelé est bien plus proche de Polyclète ${ }^{24}$ : pourtant le corps est très peu épais d'avant en arrière, d'où une

(20) W. B. EMERy, The Royal Tombs of Ballana and Qustul (Service des antiquités de l'Egypte. Mission archéologique de Nubie, 1929-1934), 1938, pp. 350 sq., n० 755, pl. 90 B. V. aussi du MEME, Nubian Treasure, 1948, p. 64, pl. 43 a, pour l'autre trépied, de construction identique mais avec une décoration figurée différente. Dans un c. $r$. de la précédente publication (Journal of the American Oriental Society, 70, 1950, pp. 193 sq.), Dows Dunham date les tombes du III au VIe sixcles de notre ère.

(21) L'objet sous la patte avant est bien un grand S, comme Bordier l'avait reconnu.

(22) V. THRAEMER, in ROSCHER, Ausführliches Lexilion der griechischen und römischen Mythologie, s. v. «Dionysos 》, vol. I, col. 1127.

(23) D'abord par Milani. Aussi, Reinach, Gazette des Beaux-Arts, sér. III, 16, 1891, IIe partie, pp. 265-276. Résumé de la littérature, Rizzo, Prassitele, pp. 117, notes aux pp. 76-78. Le bronze est dans de Ridder, Bronzes antiques du Louvre, I, p. 35, n 189, pl. 20.

(24) Les Dionysos de l'époque de Polyclète et les Bacchus apparentés par le style sont d'un 
extrême frontalité et la réduction du contraste entre la jambe portante et la jambe libre: la rigidité même que Polyclète s'efforçait de rompre semble être en voie de réapparaître.

\section{La technique.}

Certaines observations sur les curieux procédés techniques employés dans la construction de cette statuette ont leur place ici, bien qu'elles ne conduisent a aucune conclusion précise. Le bronze jaune, un alliage, probablement, contenant du zinc, a été inventé vers le milieu du Irr siècle av. J.-G. si l'on en juge par la composilion des monnaies, et fut généralement employé sous l'Empire ${ }^{25}$. Le placage d'argent partiel, obtenu avec une feuille d'argent appliquée sur le bronze par en-dessous, mais non fondue avec lui, n'a pas de parallèle exact; pourtant, on pourrait citer deux statuettes recouvertes complètement de cette façon, un Bacchus et un Apollon ${ }^{26}$. Et, sur une statuette en bronze de Cologne, une enveloppe de cuivre rouge a été martelée sur du bronze jaune, croyons-nous, par le même procédé ${ }^{26}$. On trouve quelque chose de semblable sur le trépied aux panthères (non publié) du Cabinet des Médailles, complètement recouvert d'une plaque d'argent martelée. On pourrait peut-être comparer aussi quelques objets de la trouvaille de Newstead, d'âge tardif, où le placage d'argent partiel est achevé au moyen de bords recourbés ou d'épingles en fer ${ }^{28}$. Quant au traitement des yeux, il ne reste pas assez de matière pour permettre un jugement: la conservation de la pupille en pierre est rare; un autre cas connu est celui de la Korè aux pupilles de diamant, de la fin de l'époque hellénistique ou du début de l'époque romaine ${ }^{2 \theta}$. L'œil serti à

type totalement différent; v. NeugebauER, Staatliche Museen zu Berlin, Die griechischen Bronzen der klassischen Zeit und des Hellenismus, 1951, p. 18.

(25) E. R. CALEY, Memoirs of the American Philosophical Society, XI, 1939, pp. 147 sq.; remarques plus générales sur cet alliage (anglais: brass), CALEY, dans le Sun de Baltimore, 11 avril, 1948; Lucas, Anci̊nt Egyptian Materials, 1934, p. 181. D’après mon expérience, cet alliage de couleur jaune est courant pour les statuettes à l'époque impériale, mais v. le c. r. de EichLER, Gnomon, 1951, p. 59.

(26) H. BLüMMER, Technologie und Terminologie, IV, 1887, p. 320; WEISELER, Nachr. Gött., 1886, p. 30 , n. 1; S. LERSCH, Verein von Alterthumsfreunden im Rheinlande, Fest-Programm zu Winckelmanns Geburtstage, 8, 1847 (publ. 1848), passim; Arch. Zeit., 1877, p. 78 and pl. 10; FREDERICH, Die in Ostdeutschland gefundenen Bronzestatuetten, Programm, Königliches Gymnasium zu Cüstrin, XXXIV, 1911-1912, p. $4 \mathrm{n}^{\circ \mathrm{s}}$ VII, 1 et VII. 2. et fig. 3; Neugebauer, Führer durch das Antiquarium, I, Bronzen, 1924, p. 47, n० 7291.

(27) F. Fremersdorf, Die Denkmäler des römischen Kölns, 1928, pl. 112.

(28) J. CURle, A Roman Frontier Post : the Fort at Newstead, 1911. pp. 162, 169.

(29) WALTERs, Catalogue of Bronzes - British Museum, 1899, p. 17, n 192, pl. I; Select Bronzes, 1915, pl. 2; pour la date, LAMB, Greek and Roman Bronzes, 1929, p. 223. 
pupille creusée est connu dès la fin du temps hellénistique ${ }^{30}$, mais il n'y a pas de raison de supposer qu'il soit passé de mode ensuite. Je crois qu'on le retrouve sur le trépied d'Industria, pour choisir un exemple déjà invoqué plus haut ${ }^{31}$.

Résumons-nous. Le Bacchus vient du Thuit. C'était le bas d'un pied de table mobile. Ce meuble nous reporte à un groupe de trépieds et de tables pliants qui couvrent une période commençant après la destruction de Pompéi et se prolonge jusqu'au ${ } V^{\theta}$ siècle de notre ère. La curieuse tête de bébé de la statuette ressemble à de nombreux éléments des mêmes tables et trépieds, et plus encore à ceux de la table de Sackrau que l'on a datée de la fin du $\mathrm{II}^{\mathrm{e}}$ siècle. L'étude du type révèle seulement que l'objet est romain. L'étude technique suggère une date tardive. Si nous nous reportons aux circonstances de la découverte, nous nous rappellerons que la tombe contenait des monnaies d' "Antonin " (Marc-Aurèle ou Antonin-le-Pieux) et de Postume( mort en 267), et un anneau octogonal tel qu'on en portait à la basse époque romaine. La statuette a été enterrée après 267.

Mais il faut souligner que la trace d'attache du dos est bien patinée, bien découpée: l'objet a donc été détaché à l'époque ancienne de son support, et cela, en toute probabilité, à dessein. En d'autres termes, d'applique de meuble il était devenu statuette, vraisemblablement pour les besoins de la sépulture. Il n'y a rien de surprenant qu'au IIr siècle on ait eu recours à ce procédé pour une nécessité funéraire: les statuettes, notamment de bonne qualité, se faisaient rares; il était plus facile d'adapter un ornemenl toul fail que de faire exécuter une nouvelle œuvre d'art. Il est possible qu'on ait alors apporté d'autres transformations à cet objet : la hampe du thyrse peut avoir été ajoutée ou enlevée, et la panthère présentée dans sa position actuelle.

En tout cas, la date tardive (fin du III ${ }^{\theta}$ siècle) réclamée pour l'ensevelissement ne l'est pas pour la fabrication de l'objet. La date de la table originale peut dépendre d'autres critères. A mon avis, ce Bacchus n'avait pas plus d'un siècle d'existence quand il fut mis en terre.

(Trad. P.-M. Duval). Dorothy K. Hill.

Curator of Ancient Art The Walters Art Gallery, Baltimore, U.S.A.

(30) NeUgebaufr, Bronzestatuette des Narkissos $187^{\text {er }}$ Winckelmannsprogramm der Arch. Gesellschaft zu Berlin), pp. 13 sq.

(31) Atti della Società di Archeologia, III, pl. XVI. 